\title{
On the Mean Field Instability of a Random Model for Disordered Superconductors
}

\author{
K. Ziegler ${ }^{\star}$ \\ Service de Physique Théorique, CEN Saclay, F-91191 Gif-sur-Yvette cedex, France
}

\begin{abstract}
A phenomenological model for a strongly disordered superconductor is considered. This is a modification of the Bardeen-Cooper-Schrieffer approach for a system with random phonon-electron interaction. We show that the instability of its mean field theory cannot be fitted by a power law with a positive exponent, in contrast to a recent result based on perturbation theory.
\end{abstract}

\section{Introduction}

Theoretical investigations of the effect of disorder in superconducting materials started soon after the invention of the famous Bardeen-Cooper-Schrieffer (BCS) theory in 1957 [1]. As expected from experiments, the presence of a weak random potential in this theory, describing weak disorder, does not alter essentially the superconducting properties [2]. In particular, the gap of the density of states, characterizing the superconductor below the transition point, is stable against a perturbation by the weak random potential [3]. More recent experiments with strongly disordered superconducting materials, exhibiting unusual properties, have motivated extending work in this field. In contrast to the earlier results, one is interested in the description of new properties probably caused by disorder. For instance, strong fluctuations of the gap width have been observed in experiments with various alloys. On the other hand, measurements at very low temperature $(T \approx 1 K)$ indicate a linearly increasing specific heat with temperature like in normal metals. Therefore, it was argued that this type of disordered superconductors is gapless.

The observation of a strongly fluctuating gap width leads to the construction of a phenomenological modification of the BCS theory [4]. Suppose that the Cooper pairs, the central objects of the BCS theory, are also the supercurrent carrying states of the disordered superconductor. Then it is natural to introduce an external field $\phi$ which couples with the Cooper pairs. As in the BCS theory, this field can be fixed by its saddle point value which, in turn, results in a

* Supported in part by the Deutsche Forschungsgemeinschaft 
self-consistent condition. However, due to the presence of randomness, this yields a complicated random functional equation. Instead of solving the equation, one chooses the field $\phi$ as a local random variable which is statistically independent on different points and independent of the other random variables of the model [4]. From the BCS theory it is known that the homogeneous field $\phi$ generates a gap of width $|\phi|$. Therefore, a locally fluctuating $\phi$ represents the fluctuating gap observed in experiments.

This phenomenological model is certainly a very crude caricature of the disordered superconductor. Nevertheless, we can hope that some qualitative features of a more subtle description occur in the simple model and vice versa. A typical example of that might be spontaneous symmetry breaking.

The model under consideration has been studied in a series of articles by Oppermann $[4,5]$. He suggested a mean field theory similar to the coherent potential approximation [6]. Perturbation theory around the homogeneous mean field solution is singular at the Fermi energy. This reflects an instability of the mean field theory. It was claimed that the singularity could be related to a power law for certain quantities like the average density of states. Using the power law as an ansatz, its exponent can be fitted within the perturbation expansion. For instance a square root singularity has been found for the average density of states of a three dimensional system [5].

The aim of this article is to show that the ansatz of a power law with a positive exponent, as found in the above mentioned work, is wrong. In particular, we find a positive average density of states at the point of instability (see Theorem 1 at the end of Sect. 2 for a precise statement).

The investigation of the average density of states turns out to be more complicated than in similar random tight-binding models like the Anderson model [7]. This is related to the fact that neither a cluster (or high-temperature or strong coupling ...) expansion nor a soluble model (like the Lloyd model [8]) exist here. In other words, the density of states has similar properties as the response function of the electric field in the Anderson model. It shares with the latter that it is a function which depends on complex energies of both half-planes $\rho(E+i \varepsilon, E-i \varepsilon)$.

Our proof of positivity consists of two parts. At first, we derive a lower bound for the local density of states at a lattice point $x$. This bound is the derivative of a "generating function" with respect to a random field at $x$, see (3.22). Integration over the random field compensates the derivation and we obtain for the unnormalized average density of states a bound which is extensive, i.e. it grows with the lattice size. This is shown in the second part. We separate for this purpose a subset $S$ of the lattice and estimate the contribution of the interaction with the remaining lattice. It goes like the surface of $S$. On the other hand, the isolated part goes like the volume of $S$. The normalization with the lattice volume yields therefore a positive expression.

The method of the second part is well-known and has been used for several problems in statistical physics. It was used by Wegner to give a lower bound for the average density of states of the Anderson model with random potential [9]. 


\section{The Model}

In a metal, superconductance can occur due to a collective behaviour of the conduction electrons. An attractive electron-electron interaction, mediated by phonons, leads to the formation of Cooper pairs. The classical model for such a system, in absence of an electromagnetic field, is given by the action of a grand canonical ensemble at inverse temperature $\beta$

$$
\begin{aligned}
A= & \sum_{s \in\{\uparrow \downarrow\}} \int_{0}^{\beta} \sum_{x \in \Lambda}\left[\bar{\psi}_{s}(x, \tau)\left(\frac{\partial}{\partial \tau}-\mu(x)\right) \psi_{s}(x, \tau)-\sum_{x^{\prime} \in \Lambda} \bar{\psi}_{s}(x, \tau) H_{0}\left(x, x^{\prime}\right) \psi_{s}\left(x^{\prime}, \tau\right)\right] d \tau \\
& -\int_{0}^{\beta} \sum_{x \in \Lambda} g(x) \bar{\psi}_{\uparrow}(x, \tau) \bar{\psi}_{\downarrow}(x, \tau) \psi_{\downarrow}(x, \tau) \psi_{\uparrow}(x, \tau) d \tau
\end{aligned}
$$

in a functional integral representation $[10] . \psi_{s}(x, \tau)$ and $\bar{\psi}_{s}(x, \tau)$ are Grassmann fields depending on the spin $s \in\{\uparrow, \downarrow\}$, the lattice site $x \in \Lambda$ and the "time" variable $\tau$. For simplicity, $\Lambda$ is chosen as a $d$-dimensional hypercubic lattice. This is a tight-binding description of the electrons in a crystal. It is equivalent to the introduction of a cut-off in the continuum theory in order to avoid ultraviolet divergences [10]. The nearest-neighbour matrix $H_{0}$ is related to the kinetic energy of the electrons and $\mu$ is the chemical potential. It is convenient to define the latter in a way such that $H_{0}$ has vanishing diagonal elements:

$$
H_{0}(x, x)=0 \text {. }
$$

In the partition function

$$
Z=\int \exp (-A) \prod_{x, s, \tau} d \psi_{s}(x, \tau) d \bar{\psi}_{s}(x, \tau)
$$

the quartic interaction can be expressed as an interaction with a real auxiliary field $\phi(x, \tau)$ using the identity

$$
\begin{aligned}
& \exp \left[g \bar{\psi}_{\uparrow}(x, \tau) \bar{\psi}_{\downarrow}(x, \tau) \psi_{\downarrow}(x, \tau) \psi_{\uparrow}(x, \tau)\right]=\pi^{-1 / 2} \int_{-\infty}^{\infty} \exp \left\{-[\phi(x, \tau)]^{2}\right. \\
& \left.+\sqrt{2 g} \phi(x, \tau)\left[\bar{\psi}_{\uparrow}(x, \tau) \bar{\psi}_{\downarrow}(x, \tau)+\psi_{\downarrow}(x, \tau) \psi_{\uparrow}(x, \tau)\right]\right\} d \phi(x, \tau) .
\end{aligned}
$$

By analogy to the BCS theory, we approximate the auxiliary field by the field $\phi(x)$; i.e., we ignore its $\tau$-dependence. This step enables us to diagonalize the action with respect to $\tau$ by means of a Fourier transformation

with

$$
\psi_{s}(x, \tau) \rightarrow \bar{\psi}_{s}(x, m)
$$

$$
\begin{aligned}
\psi_{s}(x, \tau) & =\beta^{-1 / 2} \sum_{m} e^{-i \xi_{m} \tau} \tilde{\psi}_{s}(x, m), \\
\bar{\psi}_{s}(x, \tau) & =\beta^{-1 / 2} \sum_{m} e^{i \xi_{m} \tau} \hat{\psi}_{s}(x, m), \\
\xi_{m} & =\pi \beta^{-1} m .
\end{aligned}
$$

and $m$ runs over all odd integers [10]. Thus, the partition function reads

$$
Z=\int \exp \left(-A^{\prime}\right) \prod_{x}\left[d \phi(x) \prod_{m, s} d \widetilde{\psi}_{s}(x, m) d \hat{\psi}_{s}(x, m)\right]
$$


with the action

$$
\begin{aligned}
A^{\prime}= & \sum_{x} \sum_{m}\left\{\sum_{s}\left[\hat{\psi}_{s}(x, m)\left[-i \xi_{m}-\mu(x)\right] \tilde{\psi}_{s}(x, m)-\sum_{x^{\prime}} \hat{\psi}_{s}(x, m) H_{0}\left(x, x^{\prime}\right) \tilde{\psi}_{s}\left(x^{\prime}, m\right)\right]\right. \\
& \left.-\sqrt{2 g(x)} \phi(x)\left[\hat{\psi}_{\uparrow}(x, m) \hat{\psi}_{\downarrow}(x,-m)+\tilde{\psi}_{\downarrow}(x, m) \tilde{\psi}_{\uparrow}(x,-m)\right]\right\}+\beta \sum_{x}[\phi(x)]^{2},
\end{aligned}
$$

which is bilinear in $\psi_{s}$. We notice that the action is not completely diagonalized by the Fourier transformation; it remains a coupling of fields with $m$ and $-m$.

This model is supposed to describe a disordered superconductor if the coupling parameter $g$ is a locally fluctuating random variable. As a second approximation, we consider

$$
\Delta(x)=\sqrt{2 g(x)} \phi(x) \quad(x \in \Lambda)
$$

as new random variables which are statistically independent of $\phi(x)$. Furthermore, $\left\{H_{0}\left(x, x^{\prime}\right)\right\}$ and $\{\mu(x)\}$ are chosen as statistically independent random variables except for the symmetry

$$
H_{0}\left(x, x^{\prime}\right)=H_{0}\left(x^{\prime}, x\right)
$$

This is the model suggested in ref. 4 . The $\phi$-integration is now entirely separated from the others in the partition function or in the Green's function

$$
\mathbf{G}\left(x, x^{\prime} ; \xi_{m}\right)=\left(\begin{array}{ll}
\left\langle\tilde{\psi}_{\uparrow}(x, m) \tilde{\psi}_{\uparrow}\left(x^{\prime}, m\right)\right\rangle & \left\langle\tilde{\psi}_{\uparrow}(x, m) \tilde{\psi}_{\downarrow}\left(x^{\prime},-m\right)\right\rangle \\
\left\langle\hat{\psi}_{\downarrow}(x,-m) \hat{\psi}_{\uparrow}\left(x^{\prime}, m\right)\right\rangle & \left\langle\hat{\psi}_{\downarrow}(x,-m) \tilde{\psi}_{\downarrow}\left(x^{\prime},-m\right)\right\rangle
\end{array}\right),
$$

and can be performed. The brackets here mean

$$
\langle\cdots\rangle=Z^{-1} \int \cdots \exp \left(-A^{\prime}\right) \prod_{x}\left[d \phi(x) \prod_{m, s} d \tilde{\psi}_{s}(x, m) d \hat{\psi}_{s}(x, m)\right] .
$$

The Green's function reads then after the $\phi$ - and $\psi$-integration as a $2|\Lambda| \times 2|\Lambda|$ matrix $(|\Lambda|$ is the number of lattice points)

$$
\mathbf{G}\left(\xi_{m}\right)=\left[\left(H_{0}+\mu\right) \sigma_{3}+\Delta \sigma_{1}+i \xi_{m} \sigma_{0}\right]^{-1}
$$

with Pauli matrices $\left\{\sigma_{j}\right\}$. The random field $\Delta$ appears in the off-diagonal elements of the $2 \times 2$ Pauli matrix structure. It will be more convenient to have the lattice non-diagonal matrix $H_{0}+\mu$ in this position. To achieve this we apply a unitary transformation to $\mathbf{G}\left(\xi_{m}\right)$ :

$$
\mathbf{G} \rightarrow \mathbf{G}^{\prime}=U_{2} \mathbf{G} U_{2}^{+}
$$

with

$$
U_{2}=2^{-1 / 2}\left(\sigma_{0}+i \sigma_{2}\right) .
$$

The new Green's function reads

$$
\mathbf{G}^{\prime}\left(\xi_{m}\right)=\left[-\left(H_{0}+\mu\right) \sigma_{1}+\Delta \sigma_{3}+i \xi_{m} \sigma_{0}\right]^{-1} .
$$

The density of states at the energy $E$ is given after the analytic continuation 
$i \xi_{m} \rightarrow E+i \varepsilon\left(\operatorname{sign} \varepsilon=\operatorname{sign} \xi_{m}\right)$ as

$$
\rho_{\Lambda}(E)=\frac{1}{\pi|\Lambda|} \lim _{\varepsilon \downarrow 0} \operatorname{Im}_{\operatorname{Tr}_{\Lambda}}\left[\mathbf{G}_{11}(-i E-\varepsilon)\right] \text {. }
$$

The diagonal blocks of the matrix (2.12)

$$
\begin{aligned}
& \mathbf{G}_{11}\left(\xi_{m}\right)=\left[H_{0}+\mu+i \xi_{m}+\Delta\left(H_{0}+\mu-i \xi_{m}\right)^{-1} \Delta\right]^{-1}, \\
& \mathbf{G}_{22}\left(\xi_{m}\right)=\left[-H_{0}-\mu+i \xi_{m}-\Delta\left(H_{0}+\mu+i \xi_{m}\right)^{-1} \Delta\right]^{-1}
\end{aligned}
$$

satisfy the relation

$$
\mathbf{G}_{22}\left(\xi_{m}\right)=-\mathbf{G}_{11}\left(-\xi_{m}\right)=-\left[\mathbf{G}_{11}\left(\xi_{m}\right)\right]^{+} .
$$

The second equation of (2.18) holds only for real $\xi_{m}$. This implies for the density of states at $E=0$ :

$$
\begin{aligned}
\rho_{\Lambda}(0) & =\frac{1}{\pi|\Lambda|} \lim _{\varepsilon \downarrow 0} \operatorname{Im} \operatorname{Tr}_{\Lambda}\left[\mathbf{G}_{11}(-\varepsilon)\right]=\frac{-i}{2 \pi|\Lambda|} \lim _{\varepsilon \downarrow 0} \operatorname{Tr}_{2 \Lambda}[\mathbf{G}(-\varepsilon)] \\
& =\frac{-i}{2 \pi|\Lambda|} \lim _{\varepsilon \downarrow 0} \operatorname{Tr}_{2 \Lambda}\left[\mathbf{G}^{\prime}(-\varepsilon)\right] .
\end{aligned}
$$

The trace operation $\operatorname{Tr}_{2 \Lambda}$ is taken with respect to the lattice sites $x$ and the Pauli matrix structure, $\operatorname{Tr}_{\Lambda}$ with respect to the lattice sites only.

The frequency-independent part of the Green's function $\mathbf{G}^{\prime}$

$$
H=-\left(H_{0}+\mu\right) \sigma_{1}+\Delta \sigma_{3}
$$

is invariant under the discrete chiral transformation

$$
H \rightarrow-\sigma_{2} H \sigma_{2} \text {, }
$$

whereas the frequency term $i \xi_{m} \sigma_{0}$ apparently breaks this symmetry. As a consequence, for the Green's function the following holds:

$$
\operatorname{Tr}_{2 \Lambda}\left[\mathbf{G}^{\prime}\left(\xi_{m}\right)\right]=\operatorname{Tr}_{2 \Lambda}\left[\sigma_{2} \mathbf{G}^{\prime}\left(\xi_{m}\right) \sigma_{2}\right]=-\operatorname{Tr}_{2 \Lambda}\left[\mathbf{G}^{\prime}\left(-\xi_{m}\right)\right] \text {. }
$$

The quantity $\operatorname{Tr}_{2 \Lambda}\left[\mathbf{G}^{\prime}\left(\xi_{m}\right)\right]$ and, therefore, $\rho_{\Lambda}(E=0)$, indicate spontaneous symmetry breaking if they do not vanish with $\xi_{m} \rightarrow 0$. The instability of the mean field theory and a possible spontaneous symmetry breaking occur at the energy $E=0$. Since we are concerned with this special problem here only $\rho_{\Lambda}(0)$ will be considered in what follows. Depending on the choice of the field $\Delta$ different situations with respect to this property can appear. There are two special realizations of the random variables which allow a direct evaluation of $\rho_{\Lambda}(E=0)$ :

a) $\Delta(x)>0$ (or $<0$ ) for all $x$ :

The trace term in (2.19) can be written

$$
\begin{aligned}
\operatorname{Tr}_{2 \Lambda}[\mathbf{G}(-\varepsilon)] & =\operatorname{Tr}_{2 \Lambda}\left\{\left[\left(H_{0}+\mu\right) \sigma_{3}+\Delta \sigma_{1}-i \varepsilon \sigma_{0}\right]^{-1}\right\} \\
& =\operatorname{Tr}_{2 \Lambda}\left\{\sigma_{3}\left[\left(H_{0}+\mu\right) \sigma_{0}-i\left(\Delta \sigma_{2}+\varepsilon \sigma_{3}\right)\right]^{-1}\right\} .
\end{aligned}
$$

The unitary transformation with

$$
U=2^{-1 / 2}\left(\sigma_{0}-i \sigma_{1}\right)
$$


diagonalizes the $\Delta$-term while the $\left(H_{0}+\mu\right)$ term remains unchanged. The right-hand side of (2.23) reads then

$$
\operatorname{Tr}_{2} \Lambda\left\{U \sigma_{3} U^{+}\left[\left(H_{0}+\mu\right) \sigma_{0}-i\left(\Delta \sigma_{3}+\varepsilon U \sigma_{3} U^{+}\right)\right]^{-1}\right\} .
$$

The limit $\varepsilon \downarrow 0$ of this expression exists, since $\Delta$ is positive (or negative):

$$
\left.\operatorname{Tr}_{2} \Lambda\left\{U \sigma_{3} U^{+}\left[\left(H_{0}+\mu\right) \sigma_{0}-i \Delta \sigma_{3}\right)\right]^{-1}\right\}
$$

and it is vanishes because of

$$
\begin{aligned}
& =\operatorname{Tr}_{2 \Lambda}\left\{\left[\left(H_{0}+\mu\right) \sigma_{3}+\Delta \sigma_{1}\right]^{-1}\right\}=\operatorname{Tr}_{2 \Lambda}\left\{U\left[\left(H_{0}+\mu\right) \sigma_{3}+\Delta \sigma_{1}\right]^{-1} U^{+}\right\} \\
& =\operatorname{Tr}_{2 \Lambda}\left\{\left[-\left(H_{0}+\mu\right) \sigma_{2}+\Delta \sigma_{1}\right]^{-1}\right\}=0 .
\end{aligned}
$$

Thus the density of states vanishes at $E=0$. It reflects the existence of a gap. This is not surprising, because it is known from the BCS theory that there is a gap of width $2|\Delta|$ if $\Delta$ is homogeneous.

b) $\mu$ is homogeneous, $\Delta$ is a staggered field:

$\Delta(x)=\bar{\Delta} D(x), \bar{\Delta}$ homogeneous, with

The transformation

$$
D(x)=(-1)^{x_{1}+x_{2}+\cdots+x_{d}}
$$

$$
\mathbf{G}(-\varepsilon) \rightarrow t \mathbf{G}(-\varepsilon) t=\mathbf{G}^{\prime \prime}(-\varepsilon)
$$

with

$$
t(x)=\left\{\begin{array}{lll}
\sigma_{0}, & \text { if } & D(x)=1 \\
\sigma_{3}, & \text { if } & D(x)=-1
\end{array}\right.
$$

yields

$$
\mathbf{G}^{\prime \prime}(-\varepsilon)=\left[\left(H_{0}-i \varepsilon\right) \sigma_{0}+\mu \sigma_{3}+\bar{\Delta} \sigma_{1}\right]^{-1} .
$$

The homogeneous term $\mu \sigma_{3}+\bar{\Delta} \sigma_{1}$ can be diagonalized by means of a global (i.e., $x$-independent) orthogonal transformation

$$
\mu \sigma_{3}+\bar{\Delta} \sigma_{1} \rightarrow \sqrt{\mu^{2}+\bar{\Delta}^{2}} \sigma_{3},
$$

such that we obtain for the trace of the Green's function

$$
\operatorname{Tr}_{2 \Lambda}[\mathbf{G}(-\varepsilon)]=\operatorname{Tr}_{2 \Lambda}\left[\mathbf{G}^{\prime \prime}(-\varepsilon)\right]=\operatorname{Tr}_{2 \Lambda}\left\{\left[\left(H_{0}-i \varepsilon\right) \sigma_{0}+\sqrt{\mu^{2}+\bar{\Delta}^{2}} \sigma_{3}\right]^{-1}\right\}
$$

This expression yields a non-vanishing density of states at $E=0$ if $\sqrt{\mu^{2}+\bar{\Delta}^{2}}$ or $-\sqrt{\mu^{2}+\bar{\Delta}^{2}}$ lies in the spectrum of $H_{0}$. Thus certain realizations of the random variables lead to a positive density of states and, therefore, to spontaneous symmetry breaking. There remains the question of whether these contribute with non-zero measure such that the average density of states is positive in the infinite volume limit. It turns out that the average density of states is indeed positive.

Theorem 1. Let $\Delta(x), \mu(x)$ and $H_{0}\left(x, x^{\prime}\right)$ be continuously distributed random variables on the interval $\left[-c, c^{\prime}\right]\left(c, c^{\prime}>0\right)$ with non-vanishing density. These random variables are statistically independent on different lattice sites $x, x^{\prime} \in \Lambda$. Then there is a positive 
average density of states

$$
\int \rho_{\Lambda}(E=0) d P\left(\left\{\Delta, \mu, H_{0}\right\}\right),
$$

which is bounded from below uniformly on $\Lambda \subset Z^{d}$.

\section{Proof of a Lower Bound on the Average Density of States}

3.1. Derivation of a Bounding Function. For any realization of the random variables the density of states, as given in (2.19), is a sum over all lattice points of the positive quantities

$$
-i\left\{\left[\mathbf{G}_{11}^{\prime}(-\varepsilon)\right]_{x x}+\left[\mathbf{G}_{22}^{\prime}(-\varepsilon)\right]_{x x}\right\}=-i\left\{\mathbf{G}_{11}^{\prime}(-\varepsilon)-\left[\mathbf{G}_{11}^{\prime}(-\varepsilon)\right]^{+}\right\}_{x x} .
$$

This is indeed positive because the Green's function can be written as

$$
\mathbf{G}_{11}^{\prime}(-\varepsilon)=(X-i \varepsilon Y)^{-1}
$$

with the Hermitian matrix

$$
X=\Delta+\left(H_{0}+\mu\right) \Delta\left(\Delta^{2}+\varepsilon^{2}\right)^{-1}\left(H_{0}+\mu\right),
$$

and the positive Hermitian matrix

$$
Y=1+\left(H_{0}+\mu\right)\left(\Delta^{2}+\varepsilon^{2}\right)^{-1}\left(H_{0}+\mu\right) .
$$

Therefore, the difference

$$
-i\left\{\mathbf{G}_{11}^{\prime}(-\varepsilon)-\left[\mathbf{G}_{11}^{\prime}(-\varepsilon)\right]^{+}\right\}=2 \varepsilon\left(X Y^{-1} X+\varepsilon^{2} Y\right)^{-1}
$$

is a positive matrix.

We may write

$$
\Theta_{\Sigma}:=2 \varepsilon \operatorname{Tr}\left[\left(X Y^{-1} X+\varepsilon^{2} Y\right)^{-1} I_{\Sigma}\right]
$$

for a finite subregion $\Sigma$ of the $d$-dimensional lattice $\Lambda$. $I_{\Sigma}$ is a projector to $\Sigma$ given by a diagonal matrix:

$$
I_{\Sigma}(x)=\left\{\begin{array}{lll}
1, & \text { if } & x \in \Sigma \\
0, & \text { if } & x \notin \Sigma
\end{array} .\right.
$$

For a sequence of disjoint subsets $\left\{\Sigma_{i}\right\}$ of $\Lambda$ we obtain a lower bound for the density of state as

$$
\rho_{\Lambda} \geqq \frac{1}{2 \pi|\Lambda|} \lim _{\varepsilon \downarrow 0} \sum_{\Sigma_{i} \subset \Lambda} \Theta_{\Sigma_{i}} .
$$

Consider now a finite cube $S$ of the lattice $\Lambda$. We restrict the fluctuations of the random variables on $S$. For any $\alpha, \alpha^{\prime}>0$ with

$$
|\Delta| \geqq \alpha
$$

and

$$
\left|H_{0}\left(x, x^{\prime}\right)\right|, \quad|\mu(x)| \leqq \alpha^{\prime}<\infty,
$$

there exists a $\tau\left(\alpha, \alpha^{\prime}\right)(1<\tau<\infty)$ with

$$
Y-Y_{S} \text { and } \tau Y_{S}-Y
$$


are positive for

$$
Y_{s}=1+\left(H_{0}+\mu\right)\left(1-I_{s}\right)\left(\Delta^{2}+\varepsilon^{2}\right)^{-1}\left(H_{0}+\mu\right)
$$

We notice that $Y_{S}$ does not depend on $\Delta(x)(x \in S)$. Then there is a lower bound for (3.6) due to the inequality (A.9) of the appendix:

$$
\Theta_{S^{\prime}} \geqq \frac{2 \varepsilon}{\tau} \operatorname{Tr}\left[\left(X Y_{S}^{-1} X+\varepsilon^{2} Y_{S}\right)^{-1} I_{S^{\prime}}\right]
$$

The subregion $S^{\prime}$ of $S$ is given as a set of lattice points which are disconnected from the lattice outside of $S$ with respect to $H_{0}$ :

$$
\left.S^{\prime}=\left\{x: x \in S ; H_{0}\left(x, x^{\prime}\right)=0, \forall x^{\prime} \in \Lambda \backslash S\right)\right\} .
$$

It is convenient to introduce now a new random variable $\mu^{\prime}$ with

$$
\mu^{\prime}(x)=\left\{\begin{array}{l}
|\hat{\Delta}(x)|^{-1} \mu(x), x \in S^{\prime} \\
\mu(x), x \notin S^{\prime}
\end{array}\right.
$$

where

$$
\hat{\Delta}(x)=\frac{\Delta^{2}(x)+\varepsilon^{2}}{\Delta(x)} .
$$

The matrix $X$ of (3.3) reads then

$$
\begin{aligned}
X= & \Delta+I_{S^{\prime}} \mu^{\prime 2} \hat{\Delta}+\left(\mathbf{1}-I_{S^{\prime}}\right) \mu^{\prime 2} \hat{\Delta}^{-1}+H_{0} \hat{\Delta}^{-1} H_{0} \\
& +I_{S^{\prime}} \mu^{\prime} T H_{0}+\left(\mathbf{1}-I_{S^{\prime}}\right) \mu^{\prime} \hat{\Delta}^{-1} H_{0}+H_{0} T \mu^{\prime} I_{S^{\prime}}+H_{0} \hat{\Delta}^{-1} \mu^{\prime}\left(1-I_{S^{\prime}}\right)
\end{aligned}
$$

with

$$
T(x)=\left\{\begin{array}{l}
\hat{\Delta}(x) /|\hat{\Delta}(x)|, x \in S^{\prime} \\
1, x \notin S^{\prime}
\end{array},\right.
$$

and we obtain by differentiation for fixed $\mu^{\prime}$ :

$$
\sum_{x \in S^{\prime}} \frac{\partial X}{\partial \Delta(x)}=I_{S^{\prime}}\left[1+\left(1-\varepsilon^{2} \Delta^{-2}\right) \mu^{\prime 2}\right]+H_{0}\left(\varepsilon^{2}-\Delta^{2}\right)\left(\Delta^{2}+\varepsilon^{2}\right)^{-2} I_{S^{\prime}} H_{0} .
$$

Therefore, we find with the function

$$
F=i \log \left[\frac{\operatorname{det}\left(X+i \varepsilon Y_{S}\right)}{\operatorname{det}\left(X-i \varepsilon Y_{S}\right)}\right]
$$

the identity

$$
\begin{aligned}
\sum_{x \in S^{\prime}} \frac{\partial F}{\partial \Delta(x)}= & i \operatorname{Tr}\left[\left(X+i \varepsilon Y_{S}\right)^{-1} \sum_{x \in S^{\prime}} \frac{\partial X}{\partial \Delta(x)}\right]-i \operatorname{Tr}\left[\left(X-i \varepsilon Y_{S}\right)^{-1} \sum_{x \in S^{\prime}} \frac{\partial X}{\partial \Delta(x)}\right] \\
= & 2 \varepsilon \operatorname{Tr}\left\{\left[\left(X Y_{S}^{-1} X+\varepsilon^{2} Y_{S}\right)^{-1}\right.\right. \\
& {\left.\left[\left(I_{S^{\prime}}+\left(1-\varepsilon^{2} \Delta^{-2}\right) \mu^{\prime 2} I_{S^{\prime}}-H_{0}\left(\Delta^{2}-\varepsilon^{2}\right)\left(\Delta^{2}+\varepsilon^{2}\right)^{-2} I_{S^{\prime}} H_{0}\right)\right]\right\} . }
\end{aligned}
$$

Since the first term on the right-hand side of (3.17) is positive and the second one 
is negative due to $|\Delta(x)|>\varepsilon$, we have

or

$$
\sum_{x \in S^{\prime}} \frac{\partial F}{\partial \Delta(x)} \leqq 2 \varepsilon \operatorname{Tr}\left[\left(X Y_{S}^{-1} X+\varepsilon^{2} Y_{S}\right)^{-1} I_{S^{\prime}}\left(1+\mu^{\prime 2}\right)\right]
$$

from (3.12).

$$
\Theta_{S^{\prime}} \geqq \frac{1}{\tau} \inf _{x \in S^{\prime}}\left(1+\mu^{\prime}(x)^{2}\right)^{-1} \sum_{x \in S^{\prime}} \frac{\partial F}{\partial \Delta(x)}
$$

The Jacobian $J$ of the substitution $\mu \rightarrow \mu^{\prime}$ is

$$
J=\prod_{x \in S^{\prime}}|\hat{\Delta}(x)| \geqq\left[1-o\left(\varepsilon^{2}\right)\right]^{\left|S^{\prime}\right|}
$$

such that we get from (3.22)

with

$$
\int \Theta_{S^{\prime}} d P\left(\left\{\Delta, \mu, H_{0}\right\}\right) \geqq \tau_{1} \int^{\prime} \sum_{x \in S^{\prime}} \frac{\partial F}{\partial \Delta(x)} d P\left(\left\{\Delta, \mu, H_{0}\right\}\right)
$$

$$
\tau_{1}=\frac{1}{\tau} \inf _{x \in S^{\prime}}\left(1+\mu^{\prime}(x)^{2}\right)^{-1}\left[1-o\left(\varepsilon^{2}\right)\right]^{\left|S^{\prime}\right|},
$$

and $\int^{\prime}$ denotes the integration with respect to the restriction of $(3.9 a, b)$. Now we divide $S^{\prime}$ into two disjoint subsets $S_{1,2}$ as

$$
S_{j}=\left\{x: x \in S^{\prime}, D(x)=(-1)^{j}\right\},
$$

where $D(x)$ is defined in (2.28). The integration over $\Delta(x)$ on $S^{\prime}$ in (3.24) can be parametrized for a fixed $x_{j} \in S_{j}$ with

as

$$
\Delta_{j}:=\Delta\left(x_{j}\right)
$$

$$
\Delta(x)=\Delta_{j}+\delta(x) \quad x_{j} \neq x \in S_{j} .
$$

The integrand in (3.24) reads then

$$
\sum_{x \in S^{\prime}} \frac{\partial F}{\partial \Delta(x)}=\sum_{j=1}^{2} \sum_{x \in S_{j}} \frac{\partial F}{\partial \Delta(x)}=\sum_{j=1}^{2} \frac{\partial F}{\partial \Delta_{j}},
$$

and we may separate the integration over $\Delta_{j}$ :

$$
\begin{aligned}
\frac{1}{\tau_{1}} \int \Theta_{S^{\prime}} d P & \geqq \sum_{j} \int_{\alpha_{j}}^{\bar{\alpha}_{j}}\left[\int_{(j)} \frac{\partial F}{\partial \Delta_{j}} \frac{d P}{d \Delta_{j}}\left(\left\{\Delta, \mu^{\prime}, H_{0}\right\}\right)\right] d \Delta_{j} \\
& \geqq \sum_{j} \int_{(j)} \int_{\alpha_{j}}^{\bar{\alpha}_{j}} \frac{\partial F}{\partial \Delta_{j}} d \Delta_{j} \inf _{\Delta_{j} \in\left[\alpha_{j}, \bar{\alpha}_{j}\right]} \frac{d P}{d \Delta_{j}}\left(\left\{\Delta, \mu^{\prime}, H_{0}\right\}\right) \\
& =\sum_{j} \int_{(j)}\left[F\left(\Delta_{j}=\bar{\alpha}_{j}\right)-F\left(\Delta_{j}=\alpha_{j}\right)\right] \inf _{\Delta_{j} \in\left[\alpha_{j}, \bar{\alpha}_{j}\right]} \frac{d P}{d \Delta_{j}}\left(\left\{\Delta, \mu^{\prime}, H_{0}\right\}\right) .
\end{aligned}
$$

The integration over $\Delta_{j}$ is restricted to

$$
\begin{array}{cl}
\alpha_{1}=\alpha, & \bar{\alpha}_{1}>\alpha, \\
\alpha_{2}<-\alpha, & \bar{\alpha}_{2}=-\alpha,
\end{array}
$$


and $\int_{(j)} \cdots d P / d \Delta_{j}$ denotes here the integration over the restricted random variables except for that of $\Delta_{j}=\Delta\left(x_{j}\right)$.

3.2. Useful Properties of the Function F. The generating $F(\{\Delta(x)\})$ of (3.18) can be generalized to the function

$$
\mathbf{F}(\{V(x)\})=i \log \operatorname{det}\left[\frac{\operatorname{det}\left(X+V+i \varepsilon Y_{S}\right)}{\operatorname{det}\left(X+V-i \varepsilon Y_{S}\right)}\right],
$$

which yields apparently

$$
\mathbf{F}(V=0)=F .
$$

This function has similar properties as the integrated density of states of a tight-binding model for a particle in a random potential $V(x)$. Wegner used this quantity to derive a lower bound for the average density of states of the random potential model [9]. We shall modify his method in order to estimate the right-hand side of (3.30). The properties of $\mathbf{F}(V)$ which are useful for the estimation are the following:

(i) $\mathbf{F}(V)$ is monotonously increasing with $V(x)(x \in \Lambda)$ :

$$
\frac{\partial \mathbf{F}(V)}{\partial V(x)}=2 \varepsilon\left[(X+V) Y_{S}^{-1}(X+V)+\varepsilon^{2} Y_{S}\right]_{x x}^{-1} \geqq 0
$$

(ii) The fluctuations of $\mathbf{F}$ with respect to $V$ are bounded:

$$
\mathbf{F}(V(x)=v)-\mathbf{F}\left(V(x)=v^{\prime}\right) \leqq 2 \pi \quad \text { for } \quad v \geqq v^{\prime}, \quad x \in \Lambda,
$$

and in particular

$$
\mathbf{F}(V(x)=\infty)-\mathbf{F}(V(x)=-\infty)=2 \pi .
$$

This holds because we can write the difference as an integral

$$
\int_{v^{\prime}}^{v} \frac{\partial \mathbf{F}}{\partial V(x)} d V(x)=i \int_{v^{\prime}}^{v}\left[\left(X+V+i \varepsilon Y_{S}\right)^{-1}-\left(X+V-i \varepsilon Y_{S}\right)^{-1}\right] d V(x) .
$$

$\left(X+V \pm i \varepsilon Y_{S}\right)_{x x}^{-1}$ as a function of $V(x)$ can be expressed in terms of a scalar function [11]

with

$$
\left.\begin{array}{l}
\left(X+V-i \varepsilon Y_{S}\right)_{x x}^{-1}=\left((z(x))^{*}+V(x)\right)^{-1} \\
\left(X+V+i \varepsilon Y_{S}\right)_{x x}^{-1}=(z(x)+V(x))^{-1}
\end{array}\right\}
$$

which implies

$$
z(x)=\left[\left(X+V-V I_{\{x\}}+i \varepsilon Y_{S}\right)_{x x}^{-1}\right]^{-1},
$$

$$
0 \leqq 2 \operatorname{Im} z(x) \int_{v^{\prime}}^{v}\left[(\operatorname{Re} z(x)+V(x))^{2}+(\operatorname{Im} z(x))^{2}\right]^{-1} d V(x) \leqq 2 \pi
$$

for the right-hand side of (3.37).

(iii) For the subset $S^{\prime \prime}$

$$
S^{\prime \prime}:=\left\{x: x \in \Lambda \backslash S, \exists x^{\prime} \in S^{\prime} \text { with } X\left(x, x^{\prime}\right) \neq 0\right\},
$$


the limit of an infinite potential on $S^{\prime \prime}$ is

$$
\lim _{V(x) \rightarrow \pm \infty\left(x \in S^{\prime \prime}\right)} \mathbf{F}(V)=\mathbf{F}_{S^{\prime}}\left(V I_{S^{\prime}}\right)+\mathbf{F}_{\Lambda \backslash\left(S^{\prime} \cup S^{\prime \prime}\right)}\left(V I_{\Lambda \backslash\left(S^{\prime} \cup S^{\prime \prime}\right)}\right) .
$$

The quantity $\mathbf{F}_{S^{\prime}}$ (and $\mathbf{F}_{\Lambda \backslash\left(S^{\prime} \cup s^{\prime \prime}\right)}$, respectively) is given by the projection of $\Lambda$ to $S^{\prime}$ :

$$
\mathbf{F}_{S^{\prime}}\left(V I_{S^{\prime}}\right)=i \log \left\{\frac{\operatorname{det}_{S^{\prime}}\left(I_{S^{\prime}}\left(X+V+i \varepsilon Y_{S}\right) I_{S^{\prime}}\right)}{\operatorname{det}_{S^{\prime}}\left(I_{S^{\prime}}\left(X+V-i \varepsilon Y_{S}\right) I_{S^{\prime}}\right)}\right\},
$$

where $\operatorname{det}_{S^{\prime}}$ is the determinant of a $\left|S^{\prime}\right| \times\left|S^{\prime}\right|$ matrix. The isolation of $\mathbf{F}$ on $S^{\prime}$ from the remaining lattice due to the limit in (3.42) can be seen directly by means of the identity

with

$$
\begin{aligned}
& \operatorname{det}\left[\left(X+V+i \varepsilon Y_{S}\right)\left(X+V-i \varepsilon Y_{S}\right)^{-1}\right] \\
& \quad=\operatorname{det}\left\{P\left(X+V+i \varepsilon Y_{S}\right) P\left[P\left(X+V-i \varepsilon Y_{S}\right) P\right]^{-1}\right\} .
\end{aligned}
$$

$$
P=\left(1-I_{S^{\prime \prime}}+V I_{S^{\prime \prime}}\right)^{-1 / 2} \text {. }
$$

The limit of an infinite potential yields then

$$
\lim _{V(x) \rightarrow \pm \infty\left(x \in S^{\prime \prime}\right)} P\left(X+V \pm i \varepsilon Y_{S}\right) P=\left(\mathbf{1}-I_{S^{\prime \prime}}\right)\left(X+V \pm i \varepsilon Y_{S}\right)\left(\mathbf{1}-I_{S^{\prime \prime}}\right)+I_{S^{\prime \prime}}
$$

From the definition of $S^{\prime \prime}$ it follows immediately that

$$
\begin{aligned}
& \left(\mathbf{1}-I_{S^{\prime \prime}}\right)\left(X+V \pm i \varepsilon Y_{S}\right)\left(\mathbf{1}-I_{S^{\prime \prime}}\right) \\
& \quad=I_{S^{\prime}}\left(X+V \pm i \varepsilon Y_{S}\right) I_{S^{\prime}}+\left(\mathbf{1}-I_{S^{\prime} \cup S^{\prime \prime}}\right)\left(X+V \pm i \varepsilon Y_{S}\right)\left(\mathbf{1}-I_{S^{\prime} \cup S^{\prime \prime}}\right),
\end{aligned}
$$

and therefore (3.42).

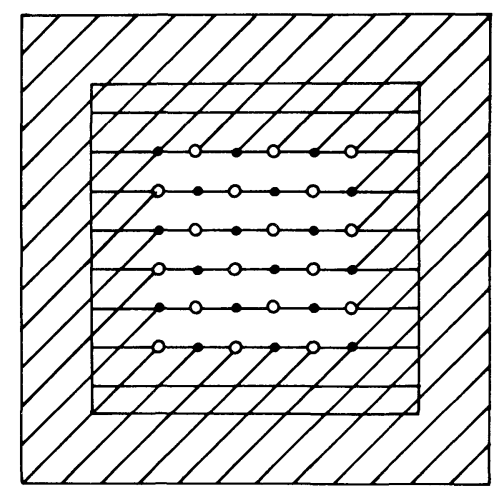

Fig. 1. The subsets $S, S^{\prime}$ and $S^{\prime \prime}: S$ is horizontally hatched and $S^{\prime \prime}$ is diagonally hatched. The internal region, which appears only horizontally hatched, is $S^{\prime}$. It consists of $S_{1}$ (points) and $S_{2}$ (circles)

(iv) Under the assumption that

$$
\begin{array}{ll}
I_{S^{\prime}}(X+V) I_{S^{\prime}} & \text { has }\left|S_{j}\right| \text { positive eigenvalues for } \Delta_{j}=d_{1}, \\
I_{S^{\prime}}(X+V) I_{S^{\prime}} & \text { has }\left|S_{j}\right| \text { negative eigenvalues for } \Delta_{j}=d_{2}
\end{array}
$$


independently of $\varepsilon$ (i.e., also in the limit $\varepsilon \downarrow 0$ ), we obtain

$$
\mathbf{F}_{S^{\prime}}\left(\Delta_{j}=d_{1}\right)-\mathbf{F}_{S^{\prime}}\left(\Delta_{j}=d_{2}\right)=\left|S_{j}\right|[2 \pi+o(\varepsilon)] .
$$

Since $I_{S^{\prime}}(X+V) I_{S^{\prime}}$ is a Hermitian matrix, there is a unitary transformation which diagonalizes this matrix and we may write

$$
\mathbf{F}_{S^{\prime}}=-\sum_{x \in S_{j}}\left[\log \left(\lambda_{x}-i \varepsilon\right)-\log \left(\lambda_{x}+i \varepsilon\right)\right]
$$

with $\left\{\lambda_{x}\right\}$ eigenvalues of $I_{S^{\prime}}(X+V) I_{S^{\prime}}$. The matrix $Y_{S}$ appears here only as $I_{S^{\prime}}$, since we have

$$
I_{S^{\prime}} Y_{S} I_{S^{\prime}}=I_{S^{\prime}}
$$

according to the definitions (3.11) and (3.13).

Then we express the left-hand side of (3.48) as an integral

$-\sum_{x \in S_{j}} \int_{\lambda_{x}\left(\Delta^{\prime}=d_{2}\right)}^{\lambda_{x}\left(\Delta^{\prime}=d_{1}\right)}\left[\left(\lambda_{x}-i \varepsilon\right)^{-1}-\left(\lambda_{x}+i \varepsilon\right)^{-1}\right] d \lambda_{x}=\sum_{x \in S_{j}}[2 \pi+o(\varepsilon)]=[2 \pi+o(\varepsilon)]\left|S_{j}\right|$,

where we have used $\lambda_{x}\left(\Delta^{\prime}=d_{1}\right)>0, \lambda_{x}\left(\Delta^{\prime}=d_{2}\right)<0$ for $x \in S_{j}$ according to the assumption. It is also clear from this argument that (3.49) is only of order $\varepsilon$ if the eigenvalues do not change the sign on the interval $\left[d_{1}, d_{2}\right]$.

Turning back to the inequality (3.30) we consider at first the difference

$$
\mathbf{F}\left(V, \Delta_{j}=\bar{\alpha}_{j}\right)-\mathbf{F}\left(V, \Delta=\alpha_{j}\right)
$$

instead of the integrand on the right-hand side of (3.30). Due to (i)-(iv) we are now in a position to estimate this expression for arbitrary values of $V(x)$. First of all, property (i) yields a lower bound if we set $V$ on $S^{\prime \prime}-\infty$ in the first term of (3.52) and $+\infty$ in the second term, respectively:

$$
\begin{aligned}
\mathbf{F}\left(V, \Delta_{j}=\bar{\alpha}_{j}\right)-\mathbf{F}\left(V, \Delta=\alpha_{j}\right) \geqq & \mathbf{F}\left(\{V(x)=-\infty\}, \Delta_{j}=\bar{\alpha}_{j}\right) \\
& -\mathbf{F}\left(\{V(x)=\infty\}, \Delta=\alpha_{j}\right) \quad\left(x \in S^{\prime \prime}\right) .
\end{aligned}
$$

After a successive application of (ii) for all $x \in S^{\prime \prime}$, the second term on the right-hand side satisfies the equation

$$
\mathbf{F}\left(\{V(x)=\infty\}, \Delta_{j}=\alpha_{j}\right)=2 \pi\left|S^{\prime \prime}\right|+\mathbf{F}\left(\{V(x)=-\infty\}, \Delta_{j}=\alpha_{j}\right) .
$$

Eventually, we find due to property (iii) that the difference in (3.52) can be expressed by the function $\mathbf{F}$ restricted to $S^{\prime}$. Thus we have

$\mathbf{F}\left(V, \Delta_{j}=\bar{\alpha}_{j}\right)-\mathbf{F}\left(V, \Delta=\alpha_{j}\right) \geqq-2 \pi\left|S^{\prime \prime}\right|+\mathbf{F}_{S^{\prime}}\left(V I_{S^{\prime}}, \Delta_{j}=\bar{\alpha}_{j}\right)-\mathbf{F}_{S^{\prime}}\left(V I_{S^{\prime}}, \Delta_{j}=\alpha_{j}\right)$

as a lower bound of (3.52). The $\mathbf{F}_{\Lambda \backslash\left(s^{\prime} \cup s^{\prime \prime}\right)}$ of (3.42) do not appear, because they are independent of $\Delta(x)(x \in S)$ and, therefore, independent of $\Delta_{j}$. Consequently, they are canceled by the difference in (3.55). Inserting this bound into (3.30), we may perform the (unrestricted) integration over the random variables on $\Lambda \backslash S$ which are 
statistically independent from those of $\mathbf{F}_{S^{\prime}}$ :

$$
\begin{gathered}
\int_{(j)}\left[\mathbf{F}\left(V, \Delta_{j}=\bar{\alpha}_{j}\right)-\mathbf{F}\left(V, \Delta_{j}=\alpha_{j}\right)\right] \inf _{\Delta_{j} \in\left[\alpha_{j}, \bar{\alpha}_{j}\right]} \frac{d P}{d \Delta_{j}} \geqq \int_{(j)}\left[-2 \pi\left|S^{\prime \prime}\right|+\mathbf{F}_{S^{\prime}}\left(V I_{S^{\prime}}, \Delta_{j}=\bar{\alpha}_{j}\right)\right. \\
\left.-\mathbf{F}_{S^{\prime}}\left(V I_{S^{\prime}}, \Delta_{j}=\alpha\right)\right] \inf _{\Delta_{j} \in\left[\alpha_{j}, \bar{\alpha}_{j}\right]} \frac{d P}{d \Delta_{j}}(\text { random variables on } S) .
\end{gathered}
$$

All manipulations hold for any value of $V$ in (3.52). In particular, we can set $V=0$ which yields an estimation of (3.30):

$$
\begin{gathered}
\sum_{j} \int_{(j)}\left[F\left(\Delta_{j}=\bar{\alpha}_{j}\right)-F\left(\Delta_{j}=\alpha_{j}\right)\right] \inf _{\Delta_{j} \in\left[\alpha_{j}, \bar{\alpha}_{j}\right]} \frac{d P}{d \Delta_{j}}\left(\left\{\Delta, \mu^{\prime}, H_{0}\right\}\right) \geqq \sum_{j} \int_{(j)}\left[-2 \pi\left|S^{\prime \prime}\right|\right. \\
\left.\left.+F_{S^{\prime}}\left(\Delta_{j}=\bar{\alpha}_{j}\right)-F_{S^{\prime}}\left(\Delta_{j}=\alpha_{j}\right)\right] \inf _{\Delta_{j} \in\left[\alpha_{j}, \bar{\alpha}_{j}\right]} \frac{d P}{d \Delta_{j}} \text { (random variables on } S\right)
\end{gathered}
$$

with $F_{S^{\prime}}=\mathbf{F}_{S^{\prime}}(V=0)$.

Our proof of $\rho_{\Lambda}(0)>0$ is accomplished when we show that the right-hand side of (3.57) is positive. Using property (iv) this means in turn that we must investigate the matrix

$I_{S^{\prime}}\left(X+i \varepsilon Y_{S}\right) I_{S^{\prime}}=I_{S^{\prime}}\left(\Delta+\mu^{\prime 2} \hat{\Delta}\right)+I_{S^{\prime}} H_{0} \hat{\Delta}^{-1} H_{0} I_{S^{\prime}}+I_{S^{\prime}}\left(\mu^{\prime} T H_{0}+H_{0} T \mu^{\prime}\right) I_{S^{\prime}}+i \varepsilon I_{S^{\prime}}$

with the parametrization

$$
\Delta(x)=\Delta_{j}+\delta(x) \quad x \in S_{j} .
$$

According to the previous discussion in Sect. 2 about a non-vanishing density of states for a staggered field $\Delta$, we suppose that $\Delta(x)$ is positive (negative) on $S_{1}\left(S_{2}\right)$. Furthermore, it is convenient for the following to choose the cube $S$ such that

$$
\left|S_{1}\right|=\left|S_{2}\right| \text {. }
$$

The determinants of $F_{S^{\prime}}\left(\Delta_{j}\right)$ in (3.18) can then be rewritten as

$$
\begin{aligned}
\operatorname{det}_{S^{\prime}}\left(I_{S^{\prime}} X I_{S^{\prime}} \pm i \varepsilon I_{S^{\prime}}\right)= & \operatorname{det}_{S_{j}}\left[I_{S_{j}} X I_{S_{j}} \pm i \varepsilon I_{S_{J}}-I_{S_{j}} X I_{S_{k}}\left(I_{S_{k}} X I_{S_{k}} \pm i \varepsilon I_{S_{k}}\right)_{S_{k}}^{-1} I_{S_{k}} X I_{S_{J}}\right] \\
& \cdot \operatorname{det}_{S_{k}}\left(I_{S_{k}} X I_{S_{k}} \pm i \varepsilon I_{S_{k}}\right) \quad(k \neq j),
\end{aligned}
$$

where $(\cdots)_{S}^{-1}$ is the inverse with respect to $S$. In particular, we have

$$
I_{S_{J}} X I_{S_{J}}=I_{S_{J}}\left(\Delta+\mu^{\prime 2} \hat{\Delta}\right)+I_{S_{J}} H_{0} \hat{\Delta}^{-1} H_{0} I_{S_{j}},
$$

and due to the fact that $H_{0}$ is a nearest-neighbour matrix we find

$$
I_{S_{J}} H_{0} \hat{\Delta}^{-1} H_{0} I_{S_{j}}=I_{S_{j}} H_{0} I_{S_{k}} \hat{\Delta}^{-1} H_{0} I_{S_{J}}
$$

with $k \neq j \in\{1,2\}$.

Moreover, the off-diagonal blocks of $X$ read

$$
I_{S_{J}} X I_{S_{k}}=I_{S_{J}}\left(\mu^{\prime} T H_{0}+H_{0} T \mu^{\prime}\right) I_{S_{k}} \quad(k \neq j) .
$$


The latter do not depend on $\Delta$ explicitly. The matrix $I_{S_{j}} H_{0} \hat{\Delta}^{-1} H_{0} I_{S}$, can be estimated by means of the lattice Laplace operator: For $j=1$ one has

$$
-I_{S_{1}} H_{0} \hat{\Delta}^{-1} H_{0} I_{S_{1}}=-I_{S_{1}} H_{0} I_{S_{2}} \hat{\Delta}^{-1} H_{0} I_{S_{1}} \geqq \inf _{x \in S_{2}}|\hat{\Delta}(x)|^{-1} I_{S_{1}} H_{0}^{2} I_{S_{1}},
$$

and analogous for $j=2(A \geqq B$ means here $A-B$ is non-negative). Then we notice that $H_{0}^{2}$ separates with $S_{1}$ and $S_{2}$ as

$$
H_{0}^{2}=I_{S_{1}} H_{0}^{2} I_{S_{1}}+I_{S_{2}} H_{0}^{2} I_{S_{2}} .
$$

This means that $H_{0}^{2}$ can be diagonalized separately by two unitary transformations $U_{j}$ :

$$
U_{j} I_{S_{j}} H_{0}^{2} I_{S_{j}} U_{j}^{+}
$$

is diagonal for an appropriate $U_{j}$. The eigenvalues correspond to those of the lattice Laplace operator if we consider constant matrix elements on $S$ :

$$
H_{0}\left(x, x^{\prime}\right)=h \quad\left(x, x^{\prime} \in S\right) .
$$

Then we may write

$$
I_{S^{\prime}} H_{0}^{2} I_{S^{\prime}} \geqq\left(I_{S^{\prime}} H_{0} I_{S^{\prime}}\right)^{2}=h^{2}\left[I_{S^{\prime}}\left(\Delta_{D}+2 d\right) I_{S^{\prime}}\right]^{2},
$$

where $-I_{S^{\prime}} \Delta_{D} I_{S^{\prime}}$ is the Dirichlet Laplace operator on $S^{\prime}$. It is positive and the eigenvalues of $\left[I_{S^{\prime}}\left(\Delta_{D}+2 d\right) I_{S^{\prime}}\right]^{2}$ have a lower bound of the order of $\left|S^{\prime}\right|^{-1 / d}$ (cf. ref. 12). Thus, $I_{S^{\prime}} H_{0}^{2} I_{S^{\prime}}$ is also positive if we allow small fluctuations for the elements of $H_{0}$ around $h$ :

$$
H_{0}\left(x, x^{\prime}\right)=h+\delta H_{0}\left(x, x^{\prime}\right) \quad\left(x, x^{\prime} \in S^{\prime}\right) .
$$

Now we can study the $\Delta$-dependence of the Hermitian part of the matrix in (3.61) for $j=1$ :

$$
I_{S_{1}} X I_{S_{1}}-I_{S_{1}} X I_{S_{2}} X I_{S_{2}}\left[\left(I_{S_{2}} X I_{S_{2}}\right)^{2}+\varepsilon^{2} I_{S_{2}}\right]_{S_{2}}^{-1} I_{S_{2}} X I_{S_{1}}
$$

with

$$
\Delta(x)>0 \quad\left(x \in S_{1}\right), \quad \Delta(x)<0 \quad\left(x \in S_{2}\right) .
$$

We suppose that the fluctuations $\delta(x)$ of $\Delta(x)$ are small compared to $\Delta_{j}$. Then we notice that $I_{S_{2}} X I_{S_{2}}$ decreases unbounded with $\mu^{\prime}(x)\left(x \in S_{2}\right)$. Thus, we may always choose this quantity such that

$$
I_{S_{2}} X I_{S_{2}}<0 \text {. }
$$

On the other hand, the second term of (3.71) is bounded for arbitrary large values of $\left(\mu^{\prime}(x)\right)^{2}\left(x \in S_{2}\right)$ while $I_{S_{1}} X I_{S_{1}}$ is independent of that. Moreover, we find that $I_{S_{1}} X I_{S_{1}}$ decreases unbounded with $\Delta(x) \uparrow 0\left(x \in S_{2}\right)$, since $I_{S_{1}} H_{0}^{2} I_{S_{1}}$ is positive. Therefore, for a given $\alpha_{1}>0$ there is an interval for $\Delta(x)\left(x \in S_{2}\right)$ such that for $\Delta_{1} \approx \alpha_{1}$ the matrix of $(3.71)$ is negative.

Furthermore, $I_{S_{1}} X I_{S_{1}}$ increases unbounded with $\Delta_{1}$, whereas the second term of (3.71) decreases (becomes more and more negative) in this case, since $I_{S_{2}} H_{0} I_{S_{1}} \hat{\Delta}^{-1} H_{0} I_{S_{2}}$ is positive and we keep $I_{S_{2}} X I_{S_{2}}$ negative by an appropriate choice of $\mu^{\prime}(x)$ on $S_{2}$. Thus, there is a sufficiently large $\bar{\alpha}_{1}>\alpha_{1}$ such that for $\Delta_{1} \approx \bar{\alpha}_{1}$ (3.71) is positive. This already would yield a positive average density of states on 
$S_{1}$. However, we can give an analogous argumentation for the contribution on $S_{2}$. We find then that

$$
I_{S_{2}} X I_{S_{2}}-I_{S_{2}} X I_{S_{1}} X I_{S_{1}}\left[\left(I_{S_{1}} X I_{S_{1}}\right)^{2}+\varepsilon^{2} I_{S_{1}}\right]_{S_{1}}^{-1} I_{S_{1}} X I_{S_{2}}
$$

is negative for $\Delta_{2} \approx \alpha_{2}$ and positive for $\Delta_{2} \approx \bar{\alpha}_{2}$ for an appropriate choice of $\alpha_{2}$ $\left(<\bar{\alpha}_{2}\right), \Delta(x)\left(x \in S_{1}\right)$ and $\mu^{\prime}(x)\left(x \in S_{1}\right)$. In both cases the appropriate choice of the variables is always possible within the conditions of Theorem 1, since the density of states obeys the scaling relation

$$
\rho_{\Lambda}\left(\left\{\Delta, \mu, H_{0}\right\}\right)=t \rho_{\Lambda}\left(\left\{t \Delta, t \mu, t H_{0}\right\}\right) ;
$$

i.e., the support of the distributions of $\Delta, \mu$, and $H_{0}$ can be extended to any finite value by a scaling transformation.

Property (iv) implies now that

$\mathbf{F}\left(V, \Delta_{j}=\bar{\alpha}_{j}\right)-\mathbf{F}\left(V, \Delta_{j}=\alpha_{j}\right)=i \log \left[\frac{\operatorname{det}\left(I_{S^{\prime}}(X+i \varepsilon) I_{S^{\prime}}\right)}{\operatorname{det}\left(I_{S^{\prime}}(X-i \varepsilon) I_{S^{\prime}}\right)}\right]=2 \pi\left(\left|S_{j}\right|-\left|S^{\prime \prime}\right|\right)+o(\varepsilon)$,

since the second determinant $\operatorname{det}\left(I_{S_{k}}(X \pm i \varepsilon) I_{S_{k}}\right)$ in (3.61) contributes only a term of order $\varepsilon$. This is due to the fact that the eigenvalues of $I_{S_{k}} X I_{S_{k}}$ do not change the sign for $\Delta_{j} \in\left[\alpha_{j}, \bar{\alpha}_{j}\right]$ (see (3.73)). Thus we conclude from (3.30) a positive lower bound for the average density of states on $S^{\prime}$ :

$$
\begin{aligned}
\int \Theta_{S^{\prime}} d P\left(\left\{\Delta, \mu, H_{0}\right\}\right) \geqq & \tau_{1} \int \inf _{\Delta_{j} \in\left[\alpha_{j}, \bar{\alpha}_{j}\right]} \frac{d P}{d \Delta_{j}}\left(\left\{\delta(x), \delta \mu^{\prime}(x), \delta H_{0}\left(x, x^{\prime}\right)\right\}_{x, x^{\prime} \in S^{\prime}}\right) \\
& \cdot 2 \pi\left[\left|S^{\prime}\right|-2\left|S^{\prime \prime}\right|+o(\varepsilon)\right],
\end{aligned}
$$

with the finite, non-vanishing perfactors defined before. $S^{\prime \prime}$ is the $d-1$ dimensional

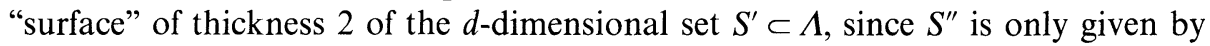
the next nearest lattice points with respect to $S^{\prime}$. Therefore, we find always a sufficiently large, finite set $S^{\prime}$ such that the last factor on the right-hand side of (3.71) is positive. This implies that the average density of states $\rho_{\Lambda}(E=0)$, defined in (2.19), is bounded from below by a positive constant uniformly in $\Lambda$.

Remark. This proof can be extended to more general matrices $H_{0}$ with finite range.

\section{Conclusion}

We have shown that the average density of states for the phenomenological model of strongly disordered superconductors is positive at $E=0$. This result has the following consequences and interpretations:

a) The particle-hole symmetry, as given in (2.21), is spontaneously broken.

b) The instability of the mean field solution cannot be fitted - in contrast to the result of a perturbation theory - by a power law with positive exponent.

c) While weak randomness does not affect the gap of the pure BCS theory, strong randomness can destroy the gap entirely.

It has been argued in ref. 4 that the model under consideration can describe a 
glass-like behaviour. In this context, a field theory, based on the replica trick similar to models of spin glasses, is considered. It would be interesting to see whether the instability of the mean field theory can be cured in terms of a perturbation theory using a "replica symmetry broken" mean field solution as suggested in the spin glass theory [13].

\section{Appendix}

For two Hermitian matrices $M_{1}, M_{2}$ the order relation

$$
M_{1}>M_{2}
$$

means that $M_{1}-M_{2}$ is a positive matrix. This relation of order can be used as the order relation of real numbers. For instance, we have for $M_{1}, M_{2}>0, M_{1}>M_{2}$ :

since

$$
M_{1}^{-1}<M_{2}^{-1}
$$

$$
\begin{aligned}
\left(M_{2}^{-1}-M_{1}^{-1}\right)^{-1} & =M_{2}\left(M_{1}-M_{2}\right)^{-1} M_{1}=M_{2}\left(M_{1}-M_{2}\right)^{-1}\left(M_{1}-M_{2}+M_{2}\right) \\
& =M_{2}+M_{2}\left(M_{1}-M_{2}\right)^{-1} M_{2}>0,
\end{aligned}
$$

and due to

Suppose that

$$
M^{-1}>0 \Leftrightarrow M>0
$$

and

$$
B, B^{\prime}>0
$$

$$
s B^{\prime}<B<t B^{\prime}
$$

with $0<s \leqq 1 \leqq t$. Then we have

$$
\frac{t}{s}\left(A B^{\prime-1} A+B^{\prime}\right)-A B^{-1} A-B=A\left(\frac{t}{s} B^{\prime-1}-B^{-1}\right) A+\frac{t}{s} B^{\prime}-B .
$$

Furthermore

$\frac{t}{s} B^{\prime-1}-B^{-1}=\frac{t-1}{s} B^{\prime-1}+\frac{1}{s}\left(B^{\prime-1}-s B^{-1}\right)=\frac{t-1}{s} B^{\prime-1}+\frac{1}{s} B^{\prime-1}\left(B-s B^{\prime}\right) B^{-1}>0$,

and

$$
\frac{t}{s} B^{\prime}-B=\left(\frac{t}{s}-t\right) B^{\prime}+t B^{\prime}-B>0,
$$

according to the assumption (A.4). Thus the expression of (A.5) is positive, which implies

$$
\left(A B^{-1} A+B\right)^{-1}>\frac{S}{t}\left(A B^{\prime-1} A+B^{\prime}\right)^{-1}
$$

due to (A.2). Finally, a positive matrix possesses positive diagonal elements such that

$$
\left[\left(A B^{-1} A+B\right)^{-1}\right]_{j j}-\frac{S}{t}\left[\left(A B^{\prime-1} A+B^{\prime}\right)^{-1}\right]_{j j}>0 .
$$




\section{References}

1. Bardeen, J., Cooper, L., Schrieffer, J. Phys. Rev. 108, 1175 (1957)

2. Anderson, P. W.: J. Phys. Chem. Sol. 11, 26 (1959)

3. Bednorz, J. G., Müller, K. A.: Z. Phys. B64, 189 (1986)

4. Oppermann, R.: Nucl. Phys. B280 (FS 18), 753 (1987)

5. Oppermann, R.: Z. Phys. B70, 49 (1988)

6. Yonezawa, F., Morigaki, K.: Suppl. Prog. Theor. Phys. 53, 1 (1973)

7. Constantinescu, F., Fröhlich, J., Spencer, T.: J. Stat. Phys. 34, 571 (1984)

8. Lloyd, J.: J. Phys. C2, 1717 (1969)

9. Wegner, F.: Z. Phys. B44, 9 (1981)

10. Sakita, B.: Quantum theory of many-variable systems and fields. Singapore: World Scientific 1985

11. Ziegler, K.: Nucl. Phys. B285 (FS 19), 606 (1987)

12. Glimm, A., Jaffe, A.: Quantum physics. Berlin, Heidelberg, New York: Springer 1981

13. Parisi, G. J.: Phys. A13, 1101 (1980)

Communicated by T. Spencer

Received November 2, 1987; in revised form July 21, 1988 
